\title{
Chiral perturbation theory and the determination of the proton radius from the muonic hydrogen Lamb shift
}

\author{
Antonio Pineda ${ }^{* \dagger}$ \\ Grup de Física Teòrica, Universitat Autònoma de Barcelona, E-08193 Bellaterra, Barcelona, \\ Spain \\ E-mail: AntonioMiguel.Pineda@uab.es
}

Recently the muonic hydrogen lamb shift has been measured with unprecedented accuracy, allowing for a precise determination of the proton radius. The error of the latter is dominated by the uncertainties associated to the hadronic corrections to the muonic hydrogen lamb shift. We show that chiral perturbation theory predicts them at leading order without any extra parameter, and in a model independent way. The reason is that these corrections are chiral enhanced.

Sixth International Conference on Quarks and Nuclear Physics

April 16-20, 2012

Ecole Polytechnique, Palaiseau, Paris

\footnotetext{
* Speaker.

$\dagger$ This work was partially supported by the spanish grants FPA2010-16963 and FPA2011-25948, and by the catalan grant SGR2009-00894.
} 


\section{Introduction}

The recent measurement of the muonic hydrogen Lamb shift, $E\left(2 P_{3 / 2}(F=2)\right)-E\left(2 S_{1 / 2}(F=\right.$ $1)$ ),

$$
E_{\text {exp }}=206.2949(32) \mathrm{meV}
$$

and the associated determination of the electromagnetic proton radius [1]:

$$
r_{p}=0.84184(67) \mathrm{fm}
$$

has led to a lot of controversy. The reason is that this number is 5 sigma away from the CODATA value, $r_{p}=0.8768(69) \mathrm{fm}$ [2]. If instead one uses this value in the theoretical expression of the muonic Hydrogen Lamb shift one gets the following discrepancy:

$$
E_{\text {exp }}-E_{t h}=0.311 \mathrm{meV}
$$

between theory and experiment. Two main options are clearly at hand: either the theoretical determination is not correct (or not as precise as claimed), or previous determinations of the proton radius were incorrect (or not as precise as claimed). Here we focus on the hadronic corrections to the muonic hydrogen Lamb shift. They produce most of the uncertainty of the determination of Ref. [1], and there are even claims that the error should be much larger, see for instance [3]. Here we would like to emphasize that in order to resolve this discrepancy, the hadronic corrections should be an order of magnitude larger than the prediction of chiral perturbation theory. Since those have been computed at leading order in the chiral counting, are model independent, and free of counterterms $[4,5,6,7]$, it is unlikely that these contributions can explain the discrepancy. We briefly review these chiral corrections here. We do it within the context of effective field theories.

The dynamics of the muonic hydrogen is characterized by several scales: $m_{p} \sim \Lambda_{\chi}$

$m_{\mu} \sim m_{\pi} \sim m_{r}=\frac{m_{\mu} m_{p}}{m_{p}+m_{\mu}}$

$m_{r} \alpha \sim m_{e}$.

By considering ratios between them the main expansion parameters are obtained:

$\frac{m_{\pi}}{m_{p}} \sim \frac{m_{\mu}}{m_{p}} \sim \frac{1}{9}$

$\frac{m_{r} \alpha}{m_{r}} \sim \frac{m_{r} \alpha^{2}}{m_{r} \alpha} \sim \alpha \sim \frac{1}{137}$.

We use the effective field theory Potential Non-Relativistic QED (pNRQED) [8]. Specially relevant for us is Ref. [4], which contains much more detailed information on the application of pNRQED to the muonic hydrogen, and we refer to it for details (see also [9, 5, 6, 7]). pNRQED profits from the hierarchy $m_{\mu} \gg m_{\mu} \alpha \gg m_{\mu} \alpha^{2}$ and the Lagrangian reads

$$
\begin{aligned}
& L_{P N R Q E D}=\int d^{3} \mathbf{r} d^{3} \mathbf{R} d t S^{\dagger}(\mathbf{r}, \mathbf{R}, t)\left\{i \partial_{0}-\frac{\mathbf{p}^{2}}{2 m_{r}}\right. \\
& \left.-V\left(\mathbf{r}, \mathbf{p}, \sigma_{1}, \sigma_{2}\right)+e \mathbf{r} \cdot \mathbf{E}(\mathbf{R}, t)\right\} S(\mathbf{r}, \mathbf{R}, t)-\int d^{3} \mathbf{r} \frac{1}{4} F_{\mu \nu} F^{\mu v}
\end{aligned}
$$


where $S$ is the field representing the muonic hydrogen, $\mathbf{R}$ the center of mass coordinate and $\mathbf{r}$ the relative distance.

$V$ stands for the potential and admits an expansion in powers of $1 / m_{\mu}$ :

$$
V\left(\mathbf{r}, \mathbf{p}, \sigma_{1}, \sigma_{2}\right)=V^{(0)}(r)+\frac{V^{(1)}(r)}{m_{\mu}}+\frac{V^{(2)}(r)}{m_{\mu}^{2}}+\cdots .
$$

The potential is obtained through matching to the underlying theory. Since pNRQED describes degrees of freedom with $E \sim m_{\mu} \alpha^{2}$, any other degree of freedom with larger energy is integrated out. This implies treating the proton and muon in a non-relativistic fashion and integrating out pions. This is the step of going from Heavy Baryon Effective Theory (HBET) [10] to NRQED [11]. By integrating out the scale $m_{\mu} \alpha$, pNRQED is obtained and the potentials appear. Schematically the path followed is the following:

$$
\operatorname{HBET}\left(m_{\pi} / m_{\mu}\right) \rightarrow \operatorname{NRQED}\left(m_{\mu} \alpha\right) \rightarrow p N R Q E D .
$$

\section{Hadronic Contributions}

We want to obtain the finite-size effects due to the hadronic structure of the proton. These effects are encoded in the coefficient multiplying the delta potential (note that the combination of NRQED matching coefficients that appears in the potential is always the same).

$$
\delta V_{\text {had }}^{(2)}(r) \equiv \frac{1}{m_{p}^{2}} D_{d}^{\text {had }} \delta^{3}(\mathbf{r}) \rightarrow \Delta E=\frac{1}{m_{p}^{2}} D_{d}^{h a d} \frac{1}{\pi}\left(\frac{m_{r} \alpha}{n}\right)^{3} \delta_{l 0}
$$

where

$$
D_{d}^{\text {had }}=-c_{3}^{\text {had }}-16 \pi \alpha d_{2}^{\text {had }}+\frac{\pi \alpha}{2} c_{D}^{\text {had }} .
$$

We define $c_{3}=c_{3}^{\text {point-like }}+c_{3}^{\text {had }}, d_{2}=d_{2}^{\text {point-like }}+d_{2}^{\text {had }} c_{D}=c_{D}^{\text {point-like }}+c_{D}^{\text {had. }}$, so that $c_{3}^{\text {had }}, d_{2}^{\text {had }}$, $c_{D}^{\text {had }}$ are the left-over of the matching coefficients of NRQED Lagrangian

$$
\delta \mathscr{L}=\cdots \frac{d_{2}}{m_{p}^{2}} F_{\mu v} D^{2} F^{\mu v}+\cdots-e \frac{c_{D}}{m_{p}^{2}} N_{p}^{\dagger} \nabla \cdot \mathbf{E} N_{p}+\cdots+\frac{c_{3}}{m_{p}^{2}} N_{p}^{\dagger} N_{p} \mu^{\dagger} \mu
$$

after subtraction of the point-like contributions. We do in this way because traditionally the pointlike contributions are already included in the "pure" QED corrections described in the previous section $^{1}$. A more extended discussion can be found in Refs. [4, 5].

$d_{2}^{\text {had }}$ encodes the hadronic vacuum polarization effect. Its contribution to the Lamb shift is tiny, $\Delta E=0.011 \mathrm{meV}$, and not much subject to uncertainty as it can be determined with enough precision from dispersion relations.

More subject to discussion are the hadronic corrections associated to $c_{3}^{\text {had }}$. They are usually split into two terms (see the discussion in Refs. $[4,5]$ ): $c_{3}^{\text {had }}=c_{3, \text { Zemach }}^{\text {had }}+c_{3, \text { hol }}^{\text {had }}$. We symbolically draw them in Figs. 1 and 2, and discuss them in the next subsections. A common feature of both of them is that they are power-like chiral enhanced: $\sim \frac{m_{\mu}}{m_{\pi}}$. This is very important, as it allows chiral perturbation theory to predict the leading order term without introducing any extra parameter. The resulting correction to the Lamb shift is of $\mathscr{O}\left(m_{\mu} \alpha^{5} \times \frac{m_{\mu}^{2}}{\Lambda_{\chi}^{2}} \times \frac{m_{\mu}}{m_{\pi}}\right)$.

\footnotetext{
${ }^{1}$ Note though, that for an strict effective theory point of view, at scales of the order of $m_{p}$, it is not a good approxima-
} 


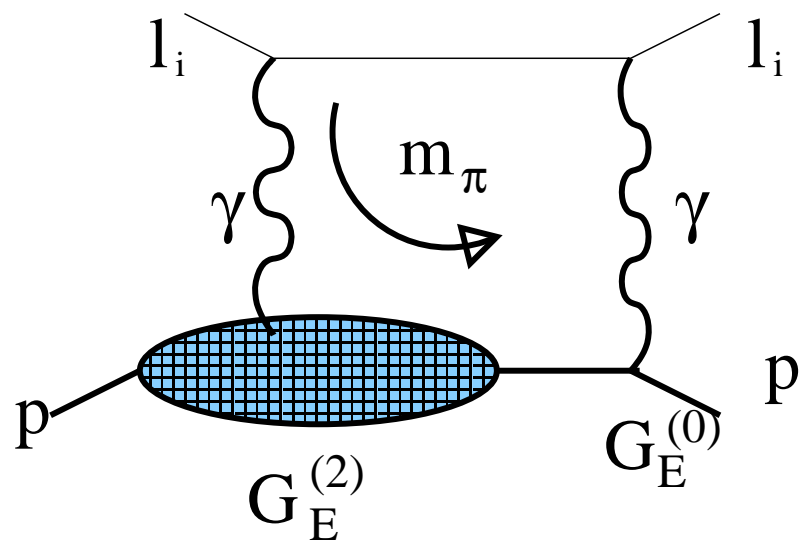

Figure 1: Symbolic representation (plus permutations) of the Zemach $\left\langle r^{3}\right\rangle$ correction, Eq. (2.4).

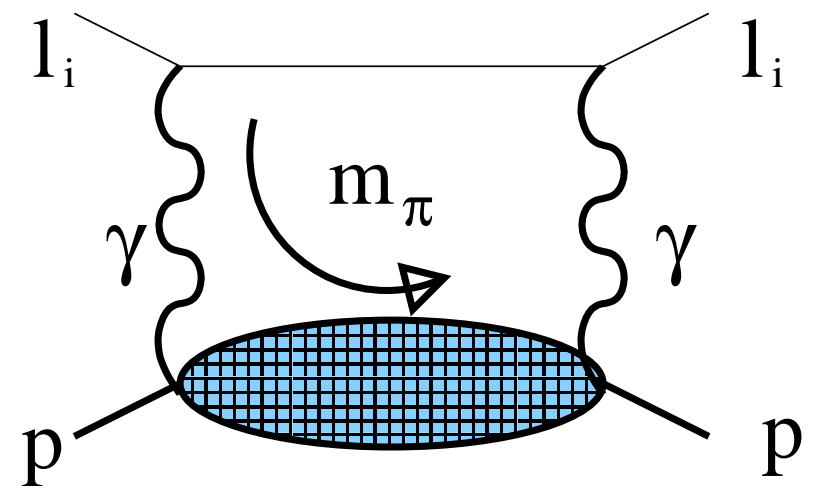

Figure 2: Symbolic representation of Eq. (2.11).

\subsection{Zemach correction, $\left\langle r^{3}\right\rangle$}

It is the one analogous to the Zemach correction defined in the hyperfine splitting [12]. It is also common to rewrite it in terms of a coefficient $\left\langle r_{p}^{3}\right\rangle$

$$
c_{3, \text { Zemach }}^{\text {had }}=\frac{\pi}{3} \alpha^{2} m_{p}^{2} m_{\mu}\left\langle r_{p}^{3}\right\rangle, \quad \frac{\left\langle r_{p}^{3}\right\rangle}{\mathrm{fm}^{3}}=\frac{96}{\pi} \int d^{D-1} k \frac{1}{\mathbf{k}^{6}} G_{E}^{(0)} G_{E}^{(2)},
$$

where $G_{E}^{(n)}$ is the electric Sachs form factor to order $n$ in the chiral counting $\left(G_{E}^{(0)}=1\right.$ and $G_{E}^{(2)}$ can be obtained from [13]). We also use dimensional regularization $(D=4+2 \varepsilon)$. This gets rid of power-like divergences which are then automatically set to zero (no need for the power-like counterterms that appear with cutoff regularization). The final result is finite and it is possible to obtain an analytic expression for the leading term in the chiral and large $N_{c}$ expansion (by including the $\Delta$ particle contribution). It reads [4]

$$
c_{3, \text { Zemach }}^{\text {had }}=2(\pi \alpha)^{2}\left(\frac{m_{p}}{4 \pi F_{0}}\right)^{2} \frac{m_{\mu}}{m_{\pi}}\left\{\frac{3}{4} g_{A}^{2}+\frac{1}{8}\right.
$$

tion to consider the proton point like. Therefore, in a way, we are introducing an "spurious" contribution in the hadronic matching coefficients. 


$$
\left.+\frac{2}{\pi} g_{\pi N \Delta}^{2} \frac{m_{\pi}}{\Delta} \sum_{r=0}^{\infty} C_{r}\left(\frac{m_{\pi}}{\Delta}\right)^{2 r}+g_{\pi N \Delta}^{2} \sum_{r=1}^{\infty} H_{r}\left(\frac{m_{\pi}}{\Delta}\right)^{2 r}\right\}
$$

where $\left(\Delta=M_{\Delta}-M_{p} \sim 300 \mathrm{MeV}\right)$

$$
\begin{gathered}
C_{r}=\frac{(-1)^{r} \Gamma(-3 / 2)}{\Gamma(r+1) \Gamma(-3 / 2-r)}\left\{B_{6+2 r}-\frac{2(r+2)}{3+2 r} B_{4+2 r}\right\}, \quad r \geq 0, \\
B_{n} \equiv \int_{0}^{\infty} d t \frac{t^{2-n}}{\sqrt{1-t^{2}}} \ln \left[\frac{1}{t}+\sqrt{\frac{1}{t^{2}}-1}\right] \quad H_{n} \equiv \frac{n !(2 n-1) ! ! \Gamma[-3 / 2]}{2(2 n) ! ! \Gamma[1 / 2+n]} .
\end{gathered}
$$

This expression produces the following number for $\left\langle r_{p}^{3}\right\rangle$ and the associated energy shift:

$$
\frac{\left.\left\langle r_{p}^{3}\right\rangle\right|_{\chi P T}}{\mathrm{fm}^{3}}=1.9 \text { (Pineda) } \rightarrow \Delta E=0.010 \frac{\left\langle r_{p}^{3}\right\rangle}{\mathrm{fm}^{3}}=0.019 \mathrm{meV}
$$

This number can be compared with some recent determinations of $\left\langle r_{p}^{3}\right\rangle$ using dispersion relations $[14,15,16,17]$

$$
\frac{\left\langle r_{p}^{3}\right\rangle \mid{ }^{e x p} "}{\mathrm{fm}^{3}}=\left\{\begin{array}{l}
2.71(13) \text { Friar }- \text { Sick } \\
2.85(8) \text { Bernauer }- \text { Arrington }
\end{array}\right\} \rightarrow \Delta E=0.027-0.029 .
$$

In principle the difference between these two determinations comes from different fit functions and data, which may give a first estimate of the associated uncertainty of the dispersion relation analysis. We find quite reassuring that the difference with the chiral computation is around $40 \%$, which could be easily accommodated with higher order corrections.

\subsection{Polarizability correction}

The determination of the polarizability correction from experiment is on more shaky grounds than for the Zemach correction, producing the larger uncertainty in the theoretical expression for the Lamb shift. The reason is that dispersion relations do not fix the result completely. The final number used in [1] was taken from the average in Ref. [18]: $\Delta E=0.015 \pm 0.004$, using the results from, [19] $\Delta E=0.012 \pm 0.002 \mathrm{meV}$, [20] $\Delta E=0.017 \pm 0.004 \mathrm{meV}$, and [21] $\Delta E=0.016 \mathrm{meV}$. For a recent discussion see Ref. [22].

Here again chiral computations may turn out to be crucial to asses the size of this correction. The reason, as before, is that the polarizability correction is power-like chiral enhanced. Therefore, chiral perturbation theory can predict the leading order term with no new parameter. This is the attitude followed in Ref. [6], where a chiral computation using dispersion relations yielded

$$
c_{3, p o l}^{\text {had }}=-e^{4} m_{p} m_{\mu} \int \frac{d^{4} k_{E}}{(2 \pi)^{4}} \frac{1}{k_{E}^{4}} \frac{1}{k_{E}^{4}+4 m_{\mu}^{2} k_{0, E}^{2}}\left\{\left(3 k_{0, E}^{2}+\mathbf{k}^{2}\right) S_{1}\left(i k_{0, E},-k_{E}^{2}\right)-\mathbf{k}^{2} S_{2}\left(i k_{0, E},-k_{E}^{2}\right)\right\}
$$

where

$$
T^{\mu v}=i \int d^{4} x e^{i q \cdot x}\left\langle p, s\left|T J^{\mu}(x) J^{v}(0)\right| p, s\right\rangle,
$$



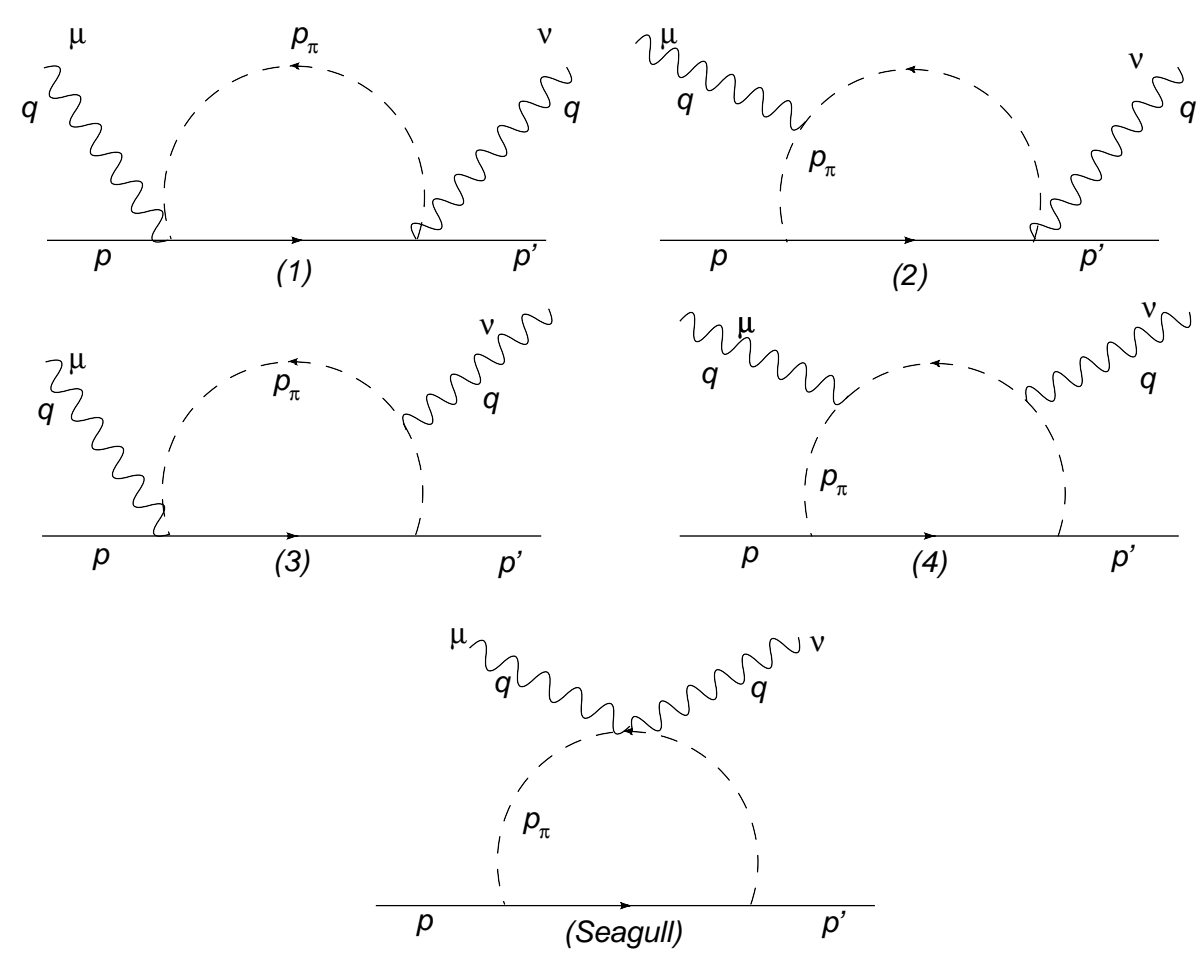

Figure 3: Diagrams contributing to $T^{i j}$ from dispersion relations. Crossed diagrams are not explicitly shown but calculated.

which has the following structure $(\rho=q \cdot p / m)$ :

$$
\begin{aligned}
T^{\mu v}= & \left(-g^{\mu v}+\frac{q^{\mu} q^{v}}{q^{2}}\right) S_{1}\left(\rho, q^{2}\right)+\frac{1}{m_{p}^{2}}\left(p^{\mu}-\frac{m_{p} \rho}{q^{2}} q^{\mu}\right)\left(p^{v}-\frac{m_{p} \rho}{q^{2}} q^{v}\right) S_{2}\left(\rho, q^{2}\right) \\
& -\frac{i}{m_{p}} \varepsilon^{\mu v \rho \sigma} q_{\rho} s_{\sigma} A_{1}\left(\rho, q^{2}\right)-\frac{i}{m_{p}^{3}} \varepsilon^{\mu v \rho \sigma} q_{\rho}\left(\left(m_{p} \rho\right) s_{\sigma}-(q \cdot s) p_{\sigma}\right) A_{2}\left(\rho, q^{2}\right)
\end{aligned}
$$

After introducing the chiral expressions for the structure factors from the diagrams in Fig. 3, one obtains

$$
\begin{aligned}
& c_{3, p o l}^{\text {had }}=-e^{4} m_{p}^{2} \frac{m_{\mu}}{m_{\pi}}\left(\frac{g_{A}}{f_{\pi}}\right)^{2} \int \frac{d^{D-1} k_{E}}{(2 \pi)^{D-1}} \frac{1}{\left(1+\mathbf{k}^{2}\right)^{4}} \int_{0}^{\infty} \frac{d w}{\pi} w^{D-5} \frac{1}{w^{2}+4 \frac{m_{\mu}^{2}}{m_{\pi}^{2}} \frac{1}{\left(1+\mathbf{k}^{2}\right)^{2}}} \\
& \times\left\{\left(2+\left(1+\mathbf{k}^{2}\right)^{2}\right) A_{E}\left(w^{2}, \mathbf{k}^{2}\right)+\left(1+\mathbf{k}^{2}\right)^{2} \mathbf{k}^{2} w^{2} B_{E}\left(w^{2}, \mathbf{k}^{2}\right)\right\}
\end{aligned}
$$

where (for $D=4$ )

$$
\begin{gathered}
A_{E}=-\frac{1}{4 \pi}\left[-\frac{3}{2}+\sqrt{1+w^{2}}+\int_{0}^{1} d x \frac{1-x}{\sqrt{1+x^{2} w^{2}+x(1-x) w^{2} \mathbf{k}^{2}}}\right], \\
B_{E}=\frac{1}{8 \pi}\left[\int_{0}^{1} d x \frac{1-2 x}{\sqrt{1+x^{2} w^{2}+x(1-x) w^{2} \mathbf{k}^{2}}}-\frac{1}{2} \int_{0}^{1} d x \frac{(1-x)(1-2 x)^{2}}{\left(1+x^{2} w^{2}+x(1-x) w^{2} \mathbf{k}^{2}\right)^{\frac{3}{2}}}\right] .
\end{gathered}
$$


This gives the number

$$
\left.\Delta E\right|_{\chi P T}(\text { pions })=0.018 \mathrm{meV} .
$$

We consider a more through chiral study of this object, in particular including the $\Delta$ particle, compulsory. The introduction of the $\Delta$ particle produced a large effect in the case of the Zemach correction, something similar may happen here. Nevertheless, we would like to emphasize that in order to explain Eq. (1.2), the corrections to the leading order chiral computation should be a factor 15 larger than the number obtained in Eq. (2.13).

\section{Conclusions}

We have briefly reviewed the determination of the hadronic corrections to the muonic hydrogen Lamb shift using chiral perturbation theory. These objects have been computed at leading order in the chiral counting, are model independent and counterterm free. Therefore, they provide with a solid determination of these parameters.

For the Zemach correction, $\left\langle r^{3}\right\rangle$, there are precise determinations obtained using dispersion relations. Chiral perturbation theory provides with a highly non-trivial double check of the magnitude of this correction. The reason is that the chiral computation is power-like chiral enhanced. It actually linearly diverges in the chiral limit. Therefore, the leading order computation in chiral perturbation theory is a pure prediction, with no free parameter. This rules out much larger values of $\left\langle r^{3}\right\rangle$ than those obtained from experiment, as such values would be in tension with the chiral perturbation theory prediction.

The polarizability correction is the major source of uncertainty. The reason is that dispersion relations alone are not able to fully determine this quantity, suffering from some ambiguity in the parameterization. Therefore, the chiral perturbation theory result may turn out to be crucial here to determine the size of this correction. Again, the chiral computation is power-like chiral enhanced and linearly diverges in the chiral limit. Thus, the leading order computation in chiral perturbation theory is a pure prediction, with no free parameter. At present there is room for improvement over the result obtained in Ref. [6] using chiral perturbation theory with dispersion relations. In particular, it does not include the contribution due to the $\Delta$ particle, which, in the case of the Zemach term, turned out to be important. It will then be very important to compute it to shrink the error associated to this correction.

\section{References}

[1] R. Pohl et al., Nature 466 (2010) 213.

[2] P. J. Mohr, B. N. Taylor and D. B. Newell, Rev. Mod. Phys. 80, 633 (2008) [arXiv:0801.0028 [physics.atom-ph]].

[3] R. J. Hill and G. Paz, Phys. Rev. Lett. 107, 160402 (2011) [arXiv:1103.4617 [hep-ph]].

[4] A. Pineda, Phys. Rev. C 71, 065205 (2005) [arXiv:hep-ph/0412142].

[5] A. Pineda, Phys. Rev. C 67, 025201 (2003) [arXiv:hep-ph/0210210]; A. Pineda, hep-ph/0308193.

[6] D. Nevado and A. Pineda, Phys. Rev. C 77, 035202 (2008) [arXiv:0712.1294 [hep-ph]]. 
[7] A. Pineda, arXiv:1108.1263 [hep-ph].

[8] A. Pineda and J. Soto, Nucl. Phys. Proc. Suppl. 64, 428 (1998) [arXiv:hep-ph/9707481].

[9] A. Pineda and J. Soto, Phys. Rev. D 59, 016005 (1999) [arXiv:hep-ph/9805424].

[10] E. E. Jenkins and A. V. Manohar, Phys. Lett. B 255, 558 (1991).

[11] W. E. Caswell and G. P. Lepage, Phys. Lett. B 167, 437 (1986).

[12] A. C. Zemach, Phys. Rev. 104, 1771 (1956).

[13] V. Bernard, H. W. Fearing, T. R. Hemmert and U. G. Meissner, Nucl. Phys. A 635, 121 (1998) [Erratum-ibid. A 642, 563 (1998)] [Nucl. Phys. A 642, 563 (1998)] [hep-ph/9801297].

[14] J. L. Friar and I. Sick, Phys. Rev. A 72, 040502 (2005) [nucl-th/0508025].

[15] M. O. Distler, J. C. Bernauer and T. Walcher, Phys. Lett. B 696, 343 (2011) [arXiv:1011.1861 [nucl-th]].

[16] J. C. Bernauer et al. [A1 Collaboration], Phys. Rev. Lett. 105, 242001 (2010) [arXiv:1007.5076 [nucl-ex]].

[17] J. Arrington, W. Melnitchouk and J. A. Tjon, Phys. Rev. C 76, 035205 (2007) [arXiv:0707.1861 [nucl-ex]].

[18] E. Borie, Phys. Rev. A 71, 032508 (2005) [physics/0410051 [physics.atom-ph]].

[19] K. Pachucki, Phys. Rev. A60, 3593 (1999).

[20] R. Rosenfelder, Phys. Lett. B 463, 317 (1999) [arXiv:hep-ph/9903352].

[21] R.N. Faustov and A.P. Martynenko, AIP Conf. Proc. 564, 277 (2001).

[22] C. E. Carlson and M. Vanderhaeghen, Phys. Rev. A 84, 020102 (2011) [arXiv:1101.5965 [hep-ph]]. 